\title{
The influence of spatial context and the role of intentionality in the interpretation of animacy from motion
}

\author{
PATRICE D. TREMOULET \\ Lockheed Martin Advanced Technology Laboratories, Cherry Hill, New Jersey \\ and \\ JACOB FELDMAN \\ Rutgers University, New Brunswick, New Jersey
}

\begin{abstract}
We present three experiments investigating how spatial context influences the attribution of animacy to a moving target. Each of our displays contained a moving object (the target) that might, depending on the way it moved, convey the impression that it was alive (animate). We investigated the mechanisms underlying this attribution by manipulating the nature of the spatial context surrounding the target. In Experiment 1, the context consisted of a simple static dot (the foil), whose position relative to the target's trajectory was manipulated. With some foil positions-for example, when the foil was lying along the path traveled by the target-animacy judgments were elevated relative to control foil locations, apparently because this context supported the impression that the target was "reacting to" or was in some other way mentally influenced by the foil. In Experiment 2, contexts consisted of a static oriented rectangle (the "paddle"). On some trials, the target collided with the paddle in a way that seemed to physically account for the target's motion pattern (in the sense of having imparted momentum to it); this condition reduced animacy ratings. Experiment 3 was similar, except that the paddles themselves were in motion; again, animacy attribution was suppressed when the target's motion seemed to have been caused by a collision with the paddle. Hence, animacy attributions can be either elevated or suppressed by the nature of the environment and the target's interaction with it. Animacy attribution tracks intentionality attribution; contrary to some earlier proposals, we conclude that attributing animacy involves, and may even require, attributing to the target some minimal mental capacity sufficient to endow the target with intentionality.
\end{abstract}

The ability to move under one's own power is one of the hallmarks of animate life. Indeed, many empirical studies demonstrate the role of motion in the interpretation of animacy. In one of the earliest such studies, Heider and Simmel (1944) presented subjects with an animation sequence featuring a disk and two triangles moving in and around a rectangular box. The observers attributed emotions and even personalities to these rigid geometric shapes, based entirely on the shapes' movements. More recent work indicates that children interpret balls and other objects as intentional, animate agents, based on motion (Dasser, Ulbaek, \& Premack, 1989; Gergely, Nádasdy, Csibra, \& Bíró, 1995; Premack, 1990) and on the contingency between motion and the child's actions (Johnson, 2000; Johnson, Booth, \& O'Hearn, 2001). Infants appear to distinguish human from nonhuman agents (Bonatti, Frot,

We are grateful to Rochel Gelman, Alan Leslie, Zenon Pylyshyn, Maggie Shiffrar, and several anonymous reviewers for helpful comments, and to NSF Grant 9875175 for support of this work. Correspondence concerning this article should be addressed to P. D. Tremoulet, Lockheed Martin Advanced Technology Laboratories, 3 Executive Campus, 6th Floor, Cherry Hill, NJ 08002 (e-mail: ptremoul@atl.lmco.com).
Zangl, \& Mehler, 2002), and recognize that humans (or human body parts) can perform actions that inanimate objects cannot (Legerstee, 1994; Leslie, 1984; Woodward, 1995; Woodward, Phillips, \& Spelke, 1993).

Several psychologists have conjectured that motion interpretation provides the basis for the concept of animacy (R. Gelman, Durgin, \& Kaufman, 1995; S. A. Gelman \& Gottfried, 1996; Leslie, 1995; Mandler, 1992; Premack \& Premack, 1995), an idea that seems plausible when one considers Michotte's (1963) classic work and other research indicating that motion gives rise to apparently "high-level" percepts. Michotte showed that temporal contingencies between two objects' changes in direction can create an impression of a causal interaction, and more recently, Schlottmann and Surian (1999) demonstrated that temporal contiguity can be sufficient to produce the percept of causation-at-a-distance. Other studies reveal that spatial contingencies between objects influence the perception of intentionality (Bassili, 1976) and that motion parameters such as speed influence the determination of intentional relations among objects (Dittrich \& Lea, 1994).

Researchers have also suggested that animacy attribution is closely related to, and perhaps even requires, the attribution of intention (Bassili, 1976; Dittrich \& Lea, 
1994; R. Gelman et al., 1995; Premack \& Premack, 1995; Stewart, 1982), a view we will refer to as the intentionality hypothesis. R. Gelman et al. (1995) offer a version of this hypothesis that features a more general claim about how motion is interpreted. They propose that it is not solely motions, but rather inferences about the causes of these motions - namely, that these causes include intentional mental states, as opposed to inanimate physical forcesthat elicit a percept of animacy. This account implies that the context surrounding a moving object can influence animacy interpretations.

The intentionality hypothesis may be contrasted with a second position in the literature, which we call the Newtonian violation hypothesis. According to this hypothesis, animacy is attributed to objects whose motion violates Newtonian laws (Stewart, 1982). Apparent violations are more strictly described as involving motions whose visible component does not conserve energy, ${ }^{1}$ suggesting the presence of a hidden energy source. Examples of such motions include accelerations and decelerations, ${ }^{2}$ as well as spontaneous stops and starts.

Some developmental data cast doubt on the Newtonian violation hypothesis. Van de Walle, Rubenstein, and Spelke (1998) report that 5- and 8-month-olds do not automatically attribute animacy to shadows that move along trajectories that are not possible paths for solid physical objects. Moreover, a series of experiments designed to test the Newtonian violation hypothesis has discredited it (Stewart, 1982). At the same time, these experiments demonstrated that certain patterns of motion do provide an impression of animacy. In particular, if an object (1) starts moving from rest, (2) changes its path so that it avoids colliding with static elements in its environment, or (3) moves in a direct path toward a static element that might be perceived as a goal, then this object is perceived to be animate (Stewart, 1982).

Most of the studies described above use complex displays involving multiple moving entities to investigate conditions that trigger high-level percepts such as intentionality. In contrast, our previous work (Tremoulet \& Feldman, 2000) has employed stimuli featuring a single particle moving in a featureless background. The particle motions were extremely simple, yet observers tended to attribute animacy to particles whose speed and direction changed simultaneously, and whose principal axes remained aligned with the direction of motion, as if they turned to align themselves with the new motion path. The larger a particle's direction change and the faster its final speed, the more animate it appeared. ${ }^{3}$ This last finding is consistent with previous research showing that slower speeds and less-direct movements are perceived as less intentional, and hence, less animate (Dittrich \& Lea, 1994).

In summary, we identify the following hypotheses from the literature:

1. Intentionality hypothesis: Animacy is perceived only when intentionality is perceived (Bassili, 1976; Dittrich \& Lea, 1994; R. Gelman et al., 1995; Stewart, 1982).

2. Newtonian violation hypothesis: Animacy is perceived whenever an object's motion path indicates that the object must have access to hidden energy sources (Bingham, Rosenblum, \& Schmidt, 1995; Stewart, 1982).

These two hypotheses have not always been clearly articulated, and have not usually been explicitly contrasted with each other, but they emerge as central themes in theoretical discussions. Under many circumstances, the hypotheses make the same predictions; however, there are also several cases in which they do not. For example, the Newtonian violation hypothesis entails that animacy judgments depend only on perceived motion paths, and do not depend on, for example, the way the agent seems to perceive or categorize its environment. In contrast, the intentionality hypothesis allows that the environment in which an action is observed may influence the observer's perception of animacy. One goal of the studies presented below is to systematically test the effect of the agent's environmental context on the perception of animacy in order to identify the specific motion/context pairings that produce an impression of animacy.

\section{EXPERIMENT 1}

In our previous studies, we methodically varied the motion trajectory to understand how it contributes to the perception of animacy. Here, we also manipulated the structure of the environment, attempting to isolate the context factors that facilitate the interpretation of animacy. In Experiment 1, we presented naive observers with simple displays showing a single white particle (referred to as the target) moving in a dark background that contained a minimal contextual cue (a static white dot, referred to as the foil). As in our previous studies, particle motions were extremely simple: constant velocity except at a single point midway through the trajectory, where speed and direction changed simultaneously. We manipulated the context by altering the position of the foil, employing positions designed to suggest different relationships between the moving particle and its environment. Three positions were intended to support intentional explanations of the velocity discontinuity, and two other positions served as controls.

\section{Method}

Subjects. Fifteen Rutgers University undergraduates participated in the experiment. All were enrolled in an Introductory Psychology class and received class credit for their participation.

Stimuli. Several computer-generated motion displays were created. Each display began with a single white particle moving in a random direction against a dark background at a constant speed of $2.84 \mathrm{~cm} / \mathrm{sec}$. After $375 \mathrm{msec}$, the particle abruptly changed both speed and direction, and continued at the new speed in the new direction for the remainder of the trial $(375 \mathrm{msec})$. The background was uniformly dark except for a small white dot (the foil). Five different environment conditions, defined by the nature of the foil's location with respect to the particle's motion trajectory, were included: prey, predator, obstacle, irrelevant, and none. (See Figure 1 for an illustration of these conditions.) In the prey condition, the foil was placed so that, after the particle changed direction, it moved directly toward the foil. In the predator condition, after the particle changed direction, it moved directly away from the foil. In the obstacle condition, the foil was placed so that the particle moved toward the foil in the first half of its trajectory but changed direction before reaching it. In the irrelevant condition, the foil was po- 
sitioned in the interior of the region defined by the acute angle formed by the two portions of the target's trajectory, clearly away from either of them. Finally, in the none condition, there was no foil.

Each display featured one of four different speed changes and one of four different direction changes. The four speed changes, defined as the ratio of initial speed to final speed, were $0.5,1.0,2.0$, and 4.0. Direction changes were either $0^{\circ}, 20^{\circ}, 40^{\circ}$, or $80^{\circ}$ to the left or to the right of the initial motion direction. Thus, there were 80 different types of displays ( 5 environment conditions $\times 4$ speed changes $\times 4$ direction changes), presented in five blocks, for a total of 400 trials. Within each block, displays were presented in a random order.

Procedure. The subjects were asked to pretend that they were classifying microscopic particles. They were seated in a dark room approximately $95 \mathrm{~cm}$ away from a monitor that was positioned face up on the floor and covered with a large cardboard ring, to simulate an oversized microscope. The subjects used a keyboard to display and rate the stimuli. They pressed 0 to initiate a trial, watched until the end, and then pressed a number between 1 and 7 to rate the degree to which the particle's motion suggested that it was alive.
Ten practice trials, randomly selected from the 80 different displays, were provided to give the subjects a sense of the types of motion patterns they would be rating. At the end of these practice trials, the subjects were invited to ask questions before beginning the experiment. Halfway through the experiment (midway through the third block), the program generating the trials paused, and the subjects were encouraged to take a break. The experimenter restarted the program whenever the subject was ready to continue. Most of the subjects took less than a minute, and none took more than a 10-min break.

Apparatus. Stimuli were generated on a Macintosh Quadra running VisionShell 3.0, and were presented on a 15-in. Macintosh monitor placed face up on the floor, so that the subjects had to look down at it. The monitor was surrounded by a cardboard "annulus" with an inner diameter of $20 \mathrm{~cm}$. The monitor and the chair that the subjects sat in were both surrounded by a heavy black curtain.

Viewed at a distance of approximately $95 \mathrm{~cm}$, the moving particles in our displays subtended visual angles of $0.123^{\circ}$ long $\times 0.054^{\circ}$ wide, and the diameters of the foils subtended $0.123^{\circ}$ visual angle. The initial speed of the moving particles was $1.71 \%$ sec.

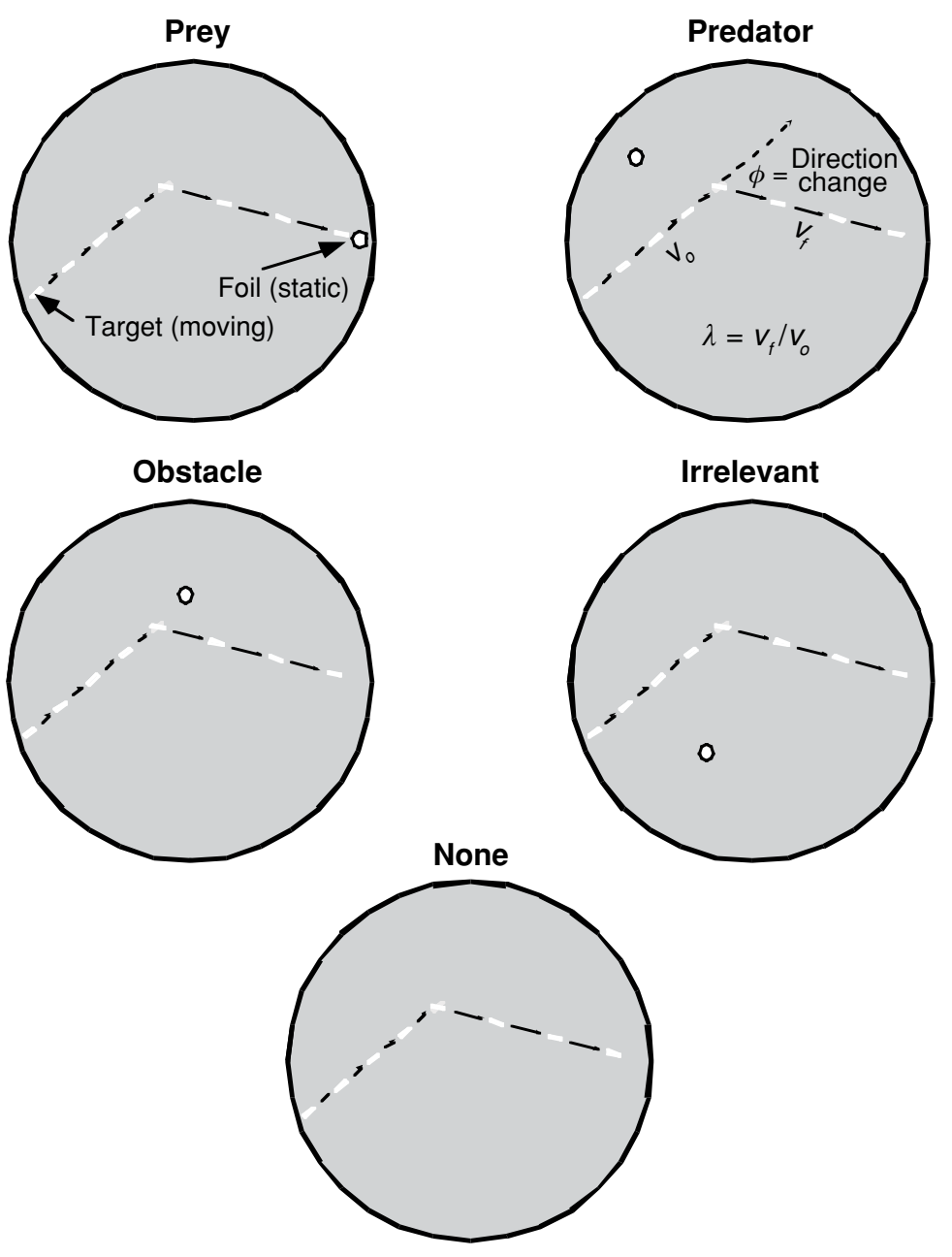

Figure 1. Five different environment (foil location) conditions. In all conditions, a rectangular particle, with aspect ratio $2: 5$, initially moves in a randomized direction at a constant speed for $375 \mathrm{msec}$, abruptly changes both speed and direction, and then continues in the new direction at the new speed for another $375 \mathrm{msec}$. In four of the five conditions, the background contains a stationary white dot (positioned as shown). In the last condition, none, no dot is present, so the background is completely featureless. 


\section{Results}

Each subject provided 400 animacy ratings. Data from all 15 subjects were combined and used to compute an ANOVA of average animacy rating, with block, environment condition, speed change, and direction change as factors.

The effects of environment condition $[F(4,56)=17.713$, $p<.001]$, direction change $[F(3,42)=14.925, p<.001]$, and speed change $[F(3,42)=21.121, p<.001]$ were significant (see Figures 2A, 2B, and 2C). Post hoc $t$ tests suggested that the environments could be divided into three groups: The prey trials were rated the most animate, followed by the predator and obstacle trials, with the irrelevant and none trials rated the lowest. Finally, animacy ratings increased with increases in speed change (increases in final speed) and with increases in direction change. The effect of block was not significant $[F(4,56)=0.285, p=.886]$.

There was also a significant interaction between speed change and direction change $[F(9,126)=5.097, p<$ $.001]$ : As speed change increased, direction changes had a weaker effect (see Figure 2D). No other two-way interactions, nor any three-way interactions, nor the four-way interaction were significant.

\section{Discussion}

Our data show that a minimal contextual cue, a static dot, can influence the degree to which a motion pattern conveys an impression of animacy. This is consistent with previous work (R. Gelman et al., 1995; Stewart, 1982) that led R. Gelman et al. to conclude that the perception of animacy does not depend solely on an object's motion path, but is also influenced by the environment. In particular, environments that facilitate intentional interpretations tend to enhance the perception of animacy. For example, our prey condition contained a stationary white dot positioned so that the particle moved toward it in the final half of its trajectory. This environment supported the interpretation that the particle wanted to reach a particular destination - namely, the location of the dot - and hence moved directly toward it in the second half of its trajectory. Such an interpretation involves attributing the source of a change in velocity to the particle's mental state(s). In contrast, in our irrelevant condition, observers might attribute to the particle a desire to reach a particular location, but there was no overt feature of the environment supporting this attribution. Thus, the perception of animacy might be weaker for irrelevant condition displays because animate explanations of the motion paths in these displays were not explicitly supported by the environment, and hence were less compelling.

Our results also indicate that simultaneous speed and direction changes tend to convey an impression of animacy whose strength increases with increases in speed and increases in direction change. This finding - and the significant two-way interaction between speed and directionreplicates our previous work using displays without foils (Tremoulet \& Feldman, 2000). The interaction between speed and direction suggests that a large change in either will produce an impression of animacy, whereas smaller

\section{A Main Effect of Environment}

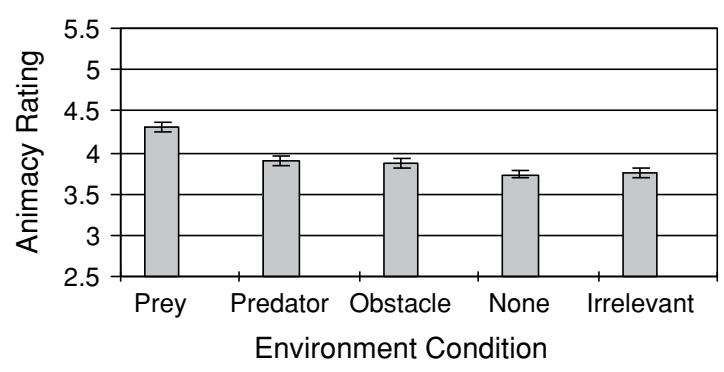

C Main Effect of Speed Change

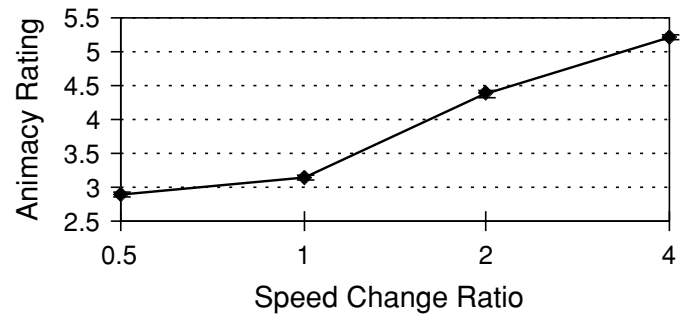

B Main Effect of Direction Change

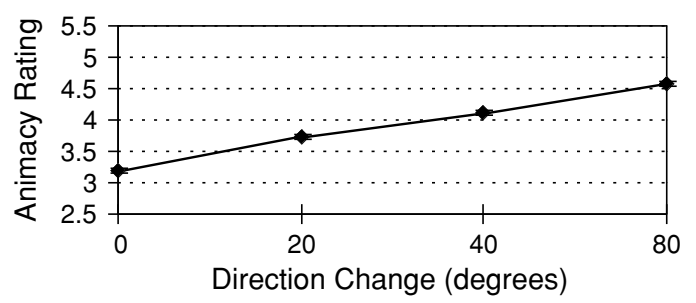

D Speed Change vs. Direction Change 2-Way Interaction

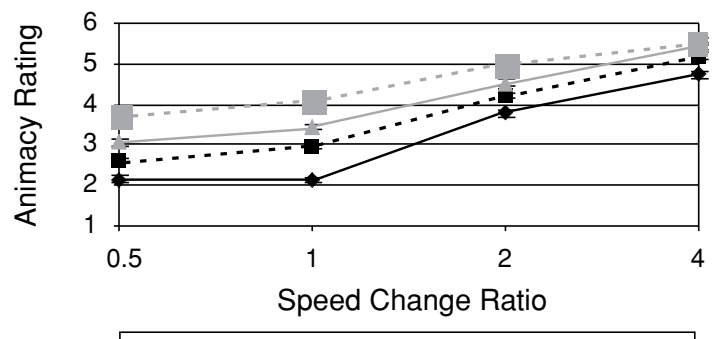

Figure 2. Significant effects found in Experiment 1: (A) Main effect of the environment condition. (B) Main effect of direction change. (C) Main effect of speed change. (D) Two-way interaction between speed change and direction change. Panel A shows that the prey condition was rated significantly higher than the predator and obstacle conditions, which, in turn, were rated significantly higher than the two control conditions (irrelevant and none). Panels $B$ and $C$ show that increasing changes in direction and increasing speed, respectively, increased animacy ratings. Panel $D$ shows that increases in direction change had less of an effect for higher speed changes (for particles with speed change ratios greater than 1). 
changes in speed (or direction) require a simultaneous change in direction (or speed) to produce similarly strong impressions of animacy.

The effect of our environment manipulation was small, relative to the effects of speed and direction change, indicating that particle motion played a more important role than context in our subjects' interpretations of animacy. ${ }^{4}$ This was also true of the R. Gelman et al. studies, which also used very simplistic environments (stationary rectangles). Thus, context may have had a small effect because our environments were so spartan (even compared with trajectories with single velocity discontinuities). However, it is also possible that context generally contributes less to the percept of animacy than motion; this would help explain why the Newtonian violation hypothesis persists in literature despite a fair amount of evidence to the contrary, and why self-starts, or other apparently internally caused accelerations, are such strong cues for animacy, even when the motivation for these motions-for example, a destination or goal - may not be obvious.

\section{EXPERIMENT 2}

In Experiment 1 displays, the moving particle was a thin rectangle that never came into contact with the stationary dot in the background. In Experiment 2 displays, the target was round, and in some cases it came into contact with the stationary rectangular "paddle" contained in the background. Previous research suggests that the subjects observing these contact displays may perceive physical causality: Michotte (1950) reported that physical causality is sometimes perceived in stimuli featuring spatial and temporal contiguity even when they involve motions that are not consistent with a purely inanimate energy transfer.

By varying the location of the paddle, we created four different environment conditions: goal, bounce, skew, and irrelevant (see Figure 3). In goal displays, the particle moved directly toward the paddle during the second half of its trajectory. This was similar to the prey condition of Experiment 1 (although in that case, the particle was rectangular and the stationary object was round). Goal displays supported the inference that the particle willfully changed velocity because it wanted to reach the paddle, so we expected subjects to give high animacy ratings to these displays. In bounce displays, the particle just touched the paddle when its direction changed, precisely the way it would during an inanimate collision with the paddle. We expected observers to infer that the particle bounced off the paddle, and thus to give low animacy ratings to these displays.

In skew displays, the particle just touched the midpoint of the paddle when its velocity changed. However, the particle's motion violated Newtonian laws governing inanimate motion; after touching the paddle, it departed in a direction $30^{\circ}$ different from the one that an inanimate
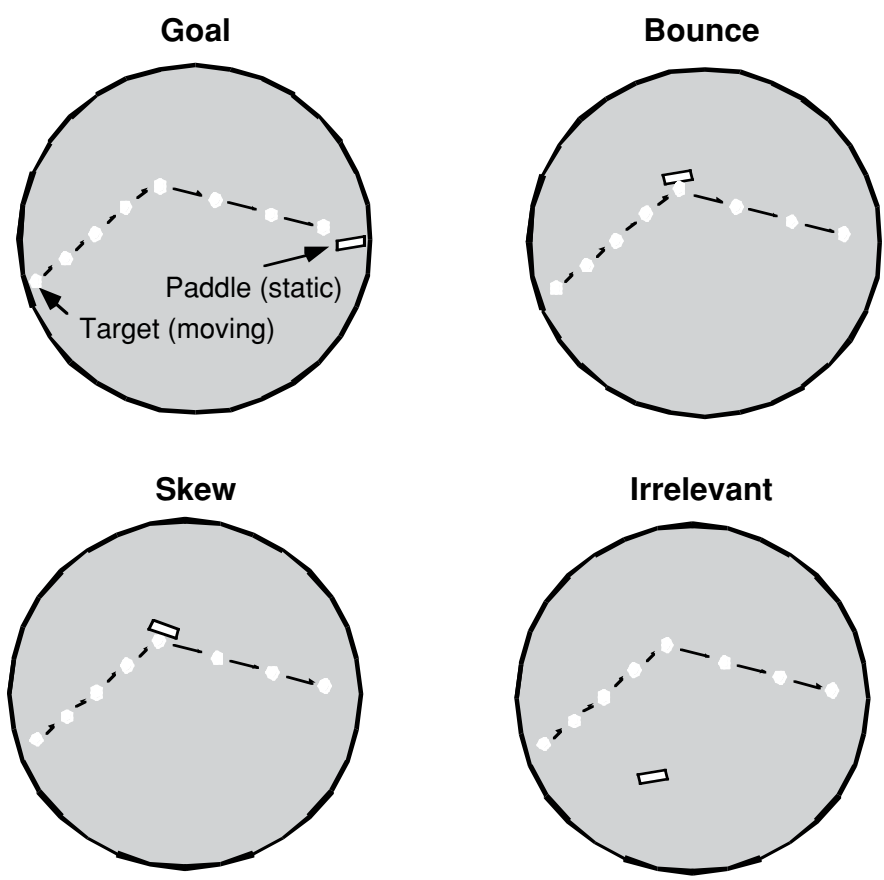

Figure 3. Four paddle conditions in Experiment 2. In all conditions, a circular particle initially moved in a randomized direction at a constant speed for 375 msec, abruptly changed both speed and direction, and then continued in the new direction at the new speed for another 375 msec. The background contained a stationary rectangular paddle (positioned as shown). 
object would have taken. ${ }^{5}$ Whereas the second half of the particle's trajectory was not consistent with an elastic collision, it is difficult to infer motivation for hurling oneself into a static object in the environment; thus, this condition represents an intermediate case, the outcome of which could not be predicted a priori. Finally, in irrelevant condition displays, the particle never touched, traveled directly toward, or traveled directly away from the paddle. (This was equivalent to Experiment 1's irrelevant condition, except the particle was round and the stationary object was rectangular.) In the irrelevant condition, the environment did not explicitly support an explanation of the particle's velocity change based on an inanimate energy transfer, nor did it support the inference that the particle suddenly changed velocity in order to approach or avoid the paddle. We hypothesized that observers would give midrange animacy ratings to irrelevant condition displays, because they would not yield as strong an impression of animacy as displays in which the paddle's location explicitly supported an intentional explanation of the particle's motion.

\section{Method}

Subjects. Twelve Rutgers University undergraduates participated in the experiment. All were enrolled in an Introductory Psychology class and received class credit for their participation. None had participated in Experiment 1.

Stimuli. Several computer-generated motion displays were created. These displays featured a round white particle moving against a dark background containing a stationary, white rectangular paddle. The particle moved in a random direction at a constant speed of $2.84 \mathrm{~cm} / \mathrm{sec}$ for $375 \mathrm{msec}$, then abruptly changed both speed and direction, and continued at the new speed in the new direction for the remainder of the trial (another $375 \mathrm{msec}$ ). Four different environment conditions, defined by the nature of the paddle's location with respect to the particle's motion trajectory, were included: goal, bounce, skew, and irrelevant.

In goal displays, the paddle was placed so that the particle traveled directly toward the midpoint of the paddle — but did not reach it - during the second half of the trajectory. The paddle was oriented so that, if it were shifted to the location where the particle changed its velocity, the angle at which the particle approached it would be equal to the angle at which it departed from it (see Figure 3A). In bounce displays, the paddle was positioned so that the particle touched its midpoint halfway through the trajectory, and the paddle was oriented so that the angle at which the particle approached was the same as the angle at which it departed (see Figure 3B). In skew displays, the center of the paddle was in the same location as it would be in bounce displays, so that the particle met the paddle's midpoint halfway through the trajectory, but the paddle's principal axis was offset by $30^{\circ}$ from the orientation it would have had in the bounce condition (see Figure 3C). Finally, in irrelevant condition displays, the paddle was positioned in the interior of the region defined by the acute angle formed by the two portions of the target's trajectory, as in the analogous condition from Experiment 1 (see Figure 3D).

Each display featured one of four different speed changes and one of four different direction changes. The four speed changes, defined as the ratio of initial speed to final speed, were $0.5,1.0,2.0$, and 4.0. Direction changes were either $0^{\circ}, 20^{\circ}, 40^{\circ}$, or $80^{\circ}$ to the left or to the right of the initial motion direction. Thus, there were 64 different types of displays (4 environment conditions $\times 4$ speed changes $\times 4$ direction changes), which were presented in five blocks, for a total of 320 trials. Within each block, displays were presented in a random order.

Viewed at a distance of approximately $95 \mathrm{~cm}$, the diameters of the moving particles in our displays subtended visual angles of $0.132^{\circ}$ and the paddles subtended visual angles of $0.264^{\circ}$ long $\times 0.054^{\circ}$ wide. The initial speed of the moving particles was $1.71^{\circ} / \mathrm{sec}$.
Procedure and Apparatus. The procedure and apparatus were the same as in Experiment 1.

\section{Results}

Each subject provided 320 animacy ratings. Data from all 12 subjects were combined and used to compute an ANOVA of average animacy rating, with block, environment condition, speed change, and direction change as factors. The main effect of block was not significant $[F(4,3520)=0.464, p=.7619]$, but the effects of environment condition $[F(3,3520)=47.379, p<.001]$ and speed change $[F(3,3520)=3.698, p=.0113]$ were. The main effect of direction change showed a trend $[F(3,3520)=$ $2.420, p=.0642]$. Figures $4 \mathrm{~A}, 4 \mathrm{~B}$, and $4 \mathrm{C}$ plot the average animacy ratings for the factors that had significant main effects. Post hoc $t$ tests indicated that the environments could be divided into two groups: The "noncontact" goal and irrelevant conditions were not significantly different from one another, but both were rated significantly higher than the "contact" bounce and skew conditions, which were also not significantly different from one another. As in previous experiments, animacy ratings increased with increases in speed change (increases in final speed).

There was also a significant interaction between environment condition and direction change $[F(9,3520)=$ $3.320, p<.001]$. For goal condition and irrelevant condition displays, animacy ratings increased with increases in the amount of direction change, but for bounce condition and skew condition displays, animacy ratings tended to decrease with increases in direction change (see Figure 4D). No other two-way interactions, nor any three-way interactions, nor the four-way interaction were significant.

\section{Discussion}

The results of Experiment 2 confirm that context can influence the impression of animacy produced by a particular motion. Specifically, the finding that bounce and skew displays were rated significantly lower than irrelevant condition displays indicates that contexts that are consistent with an object passively participating in a collision may effectively deter intentional interpretations of that object's motion path. However, the finding that goal displays were not rated significantly higher than irrelevant condition displays was unexpected, since the former, but not the latter, explicitly supports an intentional explanation of the particle's velocity change, and this difference was sufficient for subjects in Experiment 1 to rate prey displays significantly higher than irrelevant condition displays. The subjects in Experiment 2 may have given lower ratings to goal displays because the moving particles were round, so there was no (visible) change in orientation when they changed direction. ${ }^{6}$ It is also possible that the subjects perceived the paddle as an obstacle to be avoided, even in goal displays, because bounce, skew, and even irrelevant condition displays encouraged this interpretation. In other words, the other trials may have contributed to a "mental set" that hindered observers from inferring that the paddle was a goal destination. ${ }^{7}$ Apparently the procedure and instructions used here, combined with the fact 

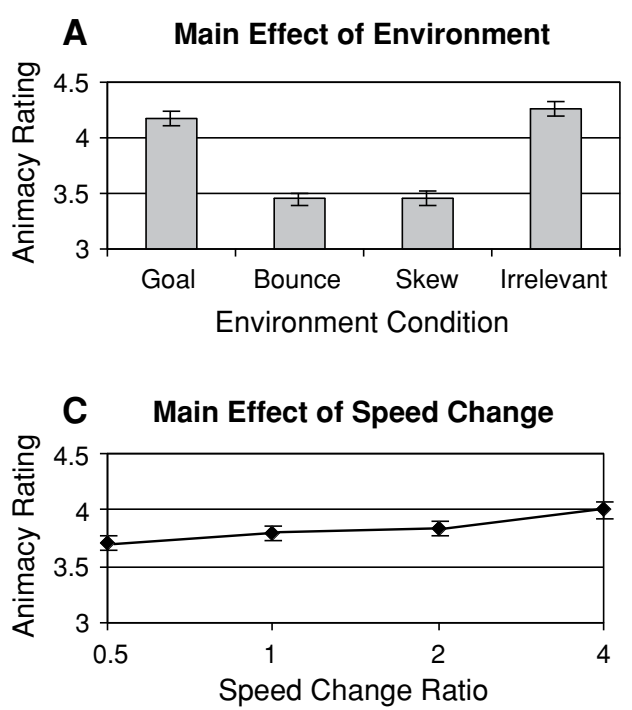

\section{B Main Effect of Direction Change}

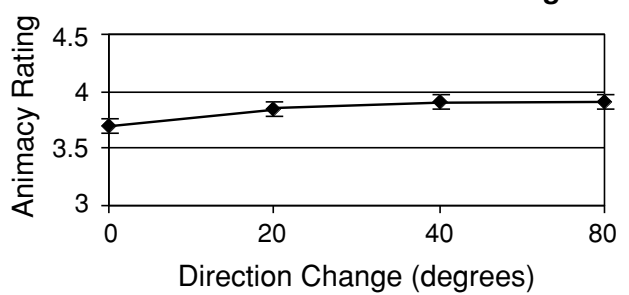

D 2-Way Interaction: Environment vs. Direction Change

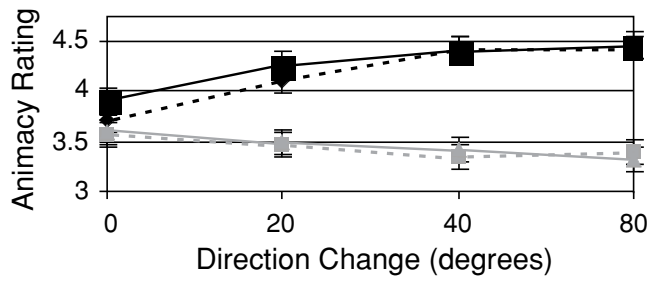

--- Goal - - - Bounce
- Skew $\longrightarrow$ Irrelevant

Figure 4. Significant effects found in Experiment 2: (A) Main effect of the environment condition. (B) Main effect of direction change. (C) Main effect of speed change. (D) Two-way interaction between environment and direction change. Panel $A$ shows that the bounce and skew conditions were rated significantly lower than the goal and irrelevant conditions. (Neither the bounce and skew conditions, nor the goal and irrelevant conditions, were rated significantly different from one another.) Panels $B$ and $C$ show that increasing changes in direction and increasing speed, respectively, increased animacy ratings. Panel $D$ shows that increases in direction change increased ratings in the goal and irrelevant conditions, but decreased ratings for the skew and bounce conditions.

that the particles were smaller than the paddles, deterred our subjects from perceiving the paddle's location to be a desirable destination for the particle.

Note that several of the bounce displays, and all of the skew displays, featured particle trajectories that were not completely consistent with an elastic collision. It is possible, even likely, that these displays were given low ratings because they were perceived to be anomalous. ${ }^{8}$ This is consistent with the idea that, when observers fail to construct simple causal explanations of a motion, they perceive that motion to be anomalous. In short, the finding that the contact (bounce and skew) displays were rated significantly lower than the irrelevant condition displays supports both the hypothesis that apparent collisions inhibit intentional explanations of velocity changes and the claim that motions are perceived to be anomalous if observers fail to infer a causal explanation that completely accounts for the perceptual data. Either way, our result is consistent with R. Gelman et al.'s (1995) causal inference theory.

Their causal inference theory can also explain why the effect of direction change differed across the two groups of environment conditions. In noncontact displays, the velocity change is attributed to the particle, so increasing the amount of direction change amounts to increasing the evidence that the particle can control its own motion, which strengthens the impression of animacy. In contrast, in contact displays, the paddle appears to cause the particle's velocity change, so increasing the amount of direction change may strengthen the impression that the particle is "passive" and hence, strengthen the impression that it is inanimate.

Unlike direction change, speed change had a consistent effect in all four environment conditions: As final speed increased, the perception of animacy increased. Although this is consistent with the results of our previous work, it is interesting to contrast the effect of speed in Experiment 2 with that of direction change. Apparently, contact between the moving target and the background object hindered subjects from formulating intentional explanations of direction changes but did not prevent them from formulating intentional explanations of accelerations. This is not especially surprising, because colliding with a static object does not cause inanimate objects to increase their speeds, so the bounce and skew environments do not suggest a straightforward mechanical explanation of a sudden acceleration by the particle. Moreover, although living creatures typically decelerate as they approach a stationary object, the "contact" trajectories in our bounce and skew displays are feasible animate motions. In fact, given the simplistic nature of our stimuli, ratings of bounce and skew displays are actually higher than one might expect; 9 one reason for this is that some subjects were initially disposed to rate all displays high "because the particles moved," a tendency we discouraged if it came out during the question period following the first 10 practice trials. Thus, the finding that overall animacy ratings increased with increases in final speed suggests that an object increasing in speed-with- 
out having obviously received energy from an external source - provides such a compelling cue for animacy that it can significantly increase ratings, even in the presence of at least one cue for inanimacy - namely, a collision with a stationary feature of the environment. At least for our spartan displays, acceleration served as the primary cue for animacy. Direction change and context may strengthen an acceleration-induced impression of animacy by helping suggest intentional explanations. The results of Experiments 1 and 2, taken together, imply that static context can enhance the effects of both speed increases and direction changes in triggering a percept of animacy, but can only inhibit the influence of direction changes.

Our third experiment investigates whether slightly altering the displays of Experiment 2, so that bounce and skew condition displays can support a purely mechanical (inanimate) explanation of an increase in a particle's speed, can reduce the effectiveness of sudden acceleration as a cue for animacy.

\section{EXPERIMENT 3}

The displays used in Experiment 2 were modified so that the paddles moved during the first half of the particle's trajectory, but abruptly stopped when the particle's veloc- ity changed. The movement of the paddle was constrained so that it always moved in a direction perpendicular to its principal axis, and when it stopped, the position of the paddle was identical to one of the paddle positions used in Experiment 2 (see Figure 5).

In Experiment 3's goal displays, the paddle traveled in the same direction that the particle would travel during the second half of its trajectory, creating the impression that the particle's velocity change was temporally and spatially contingent on the paddle's movement. Such contingencies tend to give rise to an impression of an interaction between the objects (Bassili, 1976), and thereby strengthen the perception of animacy produced by a motion display (Dittrich $\&$ Lea, 1994). Thus, these displays were expected to receive relatively high ratings. A more intuitive explanation is that the new goal displays provide an impression that the particle is following or chasing after the paddle, making the particle appear to be animate.

In the new bounce and skew displays, the movement of the paddle (more accurately, its abrupt stop) is consistent with a purely mechanical explanation of the particle suddenly increasing in speed: Upon impact, the paddle transfers some of its kinetic energy to the particle. Thus, ratings were not necessarily expected to increase with increases in speed change in these conditions. Regardless
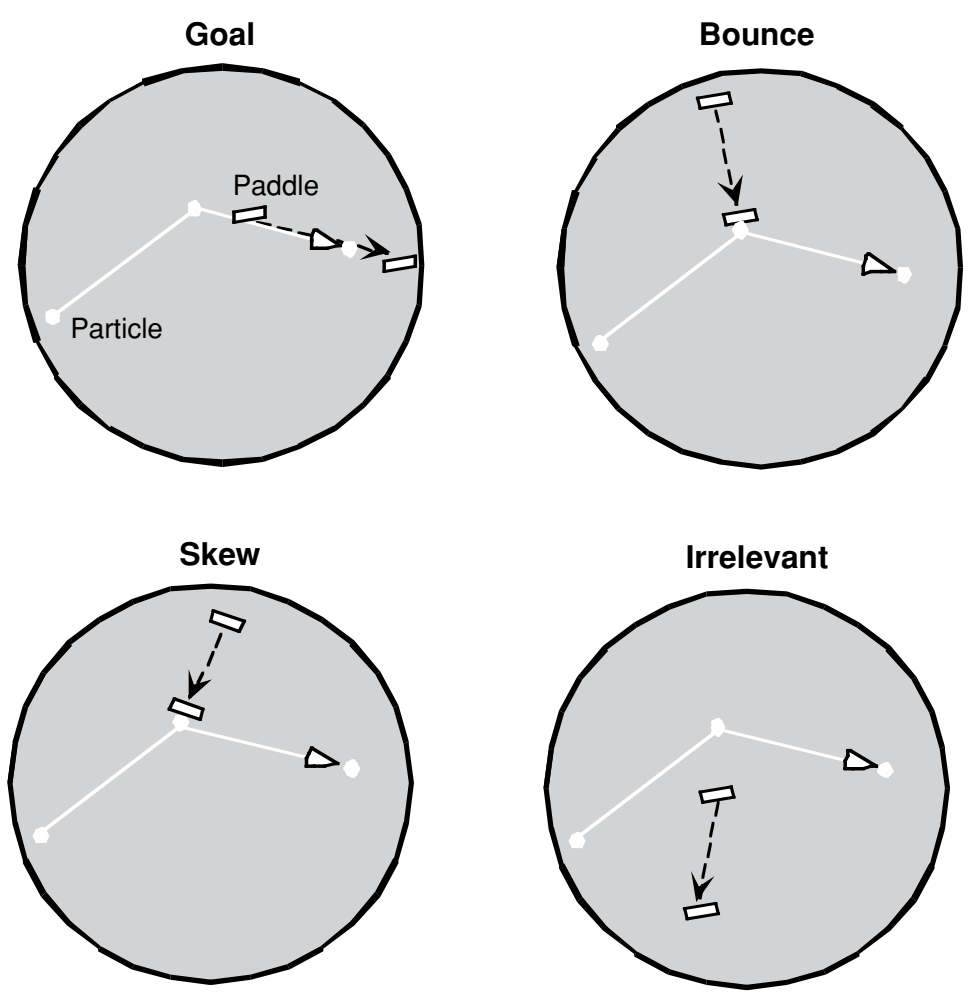

Figure 5. Four paddle conditions in Experiment 3. All conditions began with a circular particle moving in a randomized direction at a constant speed while a rectangular particle moved in another direction at twice the particle's speed. After 375 msec, the paddle stopped and the particle changed both speed and direction, continuing in the new direction at the new speed for another $375 \mathrm{msec}$. The relationship between the motion path of the rectangular paddle and the motion path of the particle varied according to condition (as shown). 
of the influence of speed changes, since bounce and skew displays provide the conditions for mechanical causality, and effectively contain a cue for inanimacy - contact consistent with a collision - these conditions were expected to receive low animacy ratings.

Finally, in the new irrelevant condition displays, the direction that the paddle traveled was not related to (that is, was neither collinear with nor parallel to) the first or second part of the particle's trajectory. However, as in all Experiment 3 displays, there was a temporal contingency that might have made the particle's motion seem more animate. On the other hand, it is unlikely that an animate entity would have simply ignored a large object moving in close proximity to it; thus, the paddle's movement may have prevented observers from inferring that the particle was animate. In short, it was unclear whether the particle trajectories in irrelevant condition displays would be perceived as animate or as anomalous; hence, it was also unclear whether these displays would be rated higher than the bounce and skew displays.

\section{Method}

Subjects. Twelve Rutgers University undergraduates participated in the experiment. All were enrolled in an Introductory Psychology class and received class credit for their participation. None had participated in Experiments 1 or 2.

Stimuli. The stimuli were very similar to those used in Experiment 2 . The only difference was that during the initial half of the particle's trajectory, the paddle in the background moved at a nonzero velocity instead of remaining stationary. Thus, each trial began with a round white particle and a white rectangular paddle, both moving at constant velocities in an otherwise uniformly dark background. The paddle moved at 1.5 times the (initial) speed of the particle. After $375 \mathrm{msec}$, the paddle abruptly stopped, and the particle changed its velocity. During the remaining $375 \mathrm{msec}$, the particle moved at a new constant velocity and the paddle was stationary. The displays were designed so that the paddle just reached a position corresponding to one of the environment conditions of Experiment 2 (goal, bounce, skew, and irrelevant) when it stopped (see Figure 5).

As in Experiment 2, each display featured one of four different speed changes (ratio of the final to the initial speed was $0.5,1.0$, 2.0 , or 4.0) and one of four different direction changes $\left(0^{\circ}, 20^{\circ}, 40^{\circ}\right.$, or $80^{\circ}$ to the left or right of the initial direction) as well as one of the four environment conditions. Hence, there were 64 different types of displays ( 4 environment conditions $\times 4$ speed changes $\times 4$ direction changes), which were presented in five blocks, for a total of 320 trials. Again, within each block, displays were presented in a random order.

Procedure and Apparatus. The procedure and apparatus were the same as in Experiments 1 and 2.

\section{Results}

Each subject provided 320 animacy ratings. Data from all 12 subjects were combined and used to compute an ANOVA of average animacy rating, with block, environment condition, speed change, and direction change as factors. All four main effects were significant: for block $[F(4,3520)=6.170, p<.001]$, for environment condition $[F(3,3520)=42.631, p<.001]$, for direction change $[F(3,3520)=4.043, p=.007]$, and for speed change $[F(3,3520)=332.780, p<.001]$ (see Figure 6). Post hoc tests revealed that animacy ratings were significantly higher during the first block than in the following four blocks, and they also indicated that, as in Experiment 2, the environments fell into two groups, with the goal and irrelevant conditions both rated significantly higher than the bounce and skew conditions. As in Experiments 1 and 2, animacy ratings increased with increases in speed change (increases in final speed) and with increases in direction change. No other interactions were significant.

\section{Discussion}

In general, the findings were consistent with the results of Experiment 2, though there were a few differences. The significant effect of block was unexpected, but is not without precedent: In previous work (Tremoulet \& Feldman, 2000), subjects' animacy ratings also decreased significantly after the first block. Analysis of individual subjects' data indicates that some of the subjects in each of our experiments significantly lowered their ratings after the first block. Thus, there seem to be individual differences in whether or not animacy ratings decline during an experimental session. However, it is unclear why the overall decline in ratings was so pronounced in Experiment 3 whereas the effect of block was not significant in Experiment 2.

The environment condition influenced ratings during Experiment 3 similarly to the way it did during Experiment 2: Bounce and skew displays were not rated significantly different from one another, and both received significantly lower ratings than goal and irrelevant condition displays. However, the difference between the latter two conditions approached significance $(p=.0546)$ this time, with the goal condition rated higher. This is not unexpected, given that Experiment 3's goal displays featured both temporal and spatial contingencies between the particle and paddle velocities, whereas its irrelevant displays featured only temporal contingencies, and that, in the absence of physical contact suggesting an inanimate energy transfer, both types of contingencies tend to increase the perception of animacy.

The third difference between the results of Experiments 2 and 3 is the effect of direction change. In Experiment 2, direction change interacted with the environment condition, whereas in Experiment 3, it did not. Post hoc tests revealed that the main effect of direction change in Experiment 3 was due to the fact that displays with $80^{\circ}$ direction changes were rated significantly higher than those with $0^{\circ}$ and $20^{\circ}$ direction changes. Thus, the effect of direction change was fairly weak (as compared with Experiment 1, in which each level of direction change was significantly different from all others). This finding is consistent with previous work (Tremoulet \& Feldman, 2000), in which we found that the effect of direction change was much weaker in displays in which the particle was a small round dot, as it was in Experiments 2 and 3, than in displays in which the particle was a small white rectangle, as it was in Experiment 1. This is because changes in direction provide more information when particles are rectangular than when they are round; if alignment also changes, the direction change provides a more compelling impression of animacy, and if alignment doesn't change, the direction change can weaken the impression of animacy. 

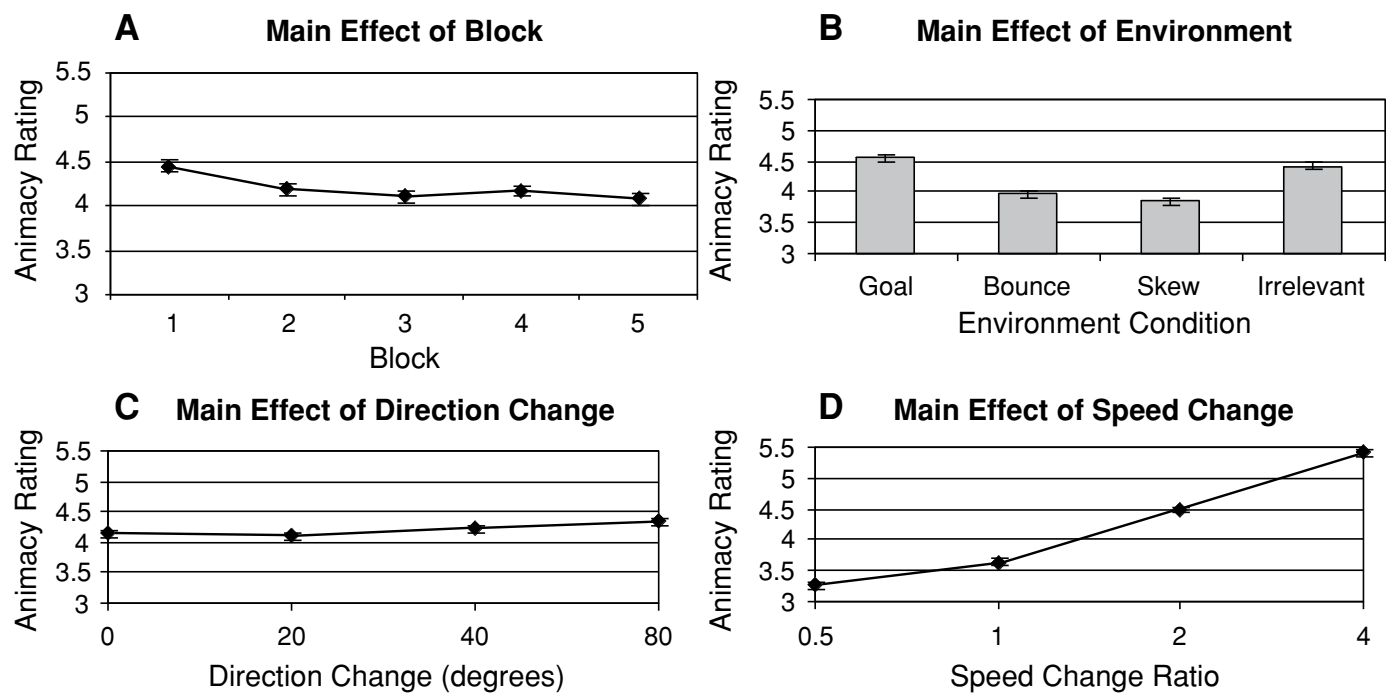

\begin{abstract}
Figure 6. Significant effects found in Experiment 3: (A) Main effect of block. (B) Main effect of environment condition. (C) Main effect of direction change. (D) Main effect of speed change. Panel A shows that animacy ratings decreased significantly after the first block. Panel B shows that the bounce and skew conditions were not rated significantly differently from one another, but they were rated significantly lower than both the goal and irrelevant conditions. Panel $\mathrm{C}$ shows that displays with $8^{\circ}$ direction changes were rated significantly higher than displays with smaller direction changes. Finally, panel $D$ shows that animacy ratings increased with increases in speed.
\end{abstract}

The effect of speed change was consistent with the results of all our previous experiments: As final speed increased, the perception of animacy increased. This pattern held across all four environment conditions, indicating that adding movement to the paddle, thereby making it plausible for it to have transferred energy to the particle, did not have the anticipated effect. The subjects continued to give higher ratings to bounce and skew displays with higher speed increases. In sum, the main effect of speed in Experiment 3 replicates previous results and offers additional evidence that a sudden acceleration serves as such a strong cue for animacy that it can influence subjects' ratings even in the presence of a contextual cue for inanimacy, such as an apparent collision. ${ }^{10}$

When the results of Experiments 2 and 3 are taken together with the results of Experiment 1, they provide strong evidence in favor of an account of the perception of animacy in which the inference of intentional mental states plays a key role. The results suggest that (1) observers automatically attempt to infer the causes of motions, (2) context can influence what types of causes are inferred, and (3) animacy is perceived if, and only if, an intentional mental state is inferred to be (one of) the cause(s) of an object's motion.

\section{GENERAL DISCUSSION}

The experiments presented here demonstrate that a slight manipulation of context-changing the location of a static object, or the direction of motion of a moving object - can significantly influence the degree to which an extremely simple motion is perceived as animate movement. Previous work had suggested that basic, uncompli- cated motions, including a start from rest (Stewart, 1982) or a single velocity discontinuity (Tremoulet \& Feldman, 2000 ), can be sufficient to suggest animacy. The experiments presented here show that the spatial context can augment or suppress this impression, depending on how it relates to the target's motion trajectory. In Experiment 1, we found that a particle's motion was more likely to be seen as animate if the motion was consistent with the particle reacting to features of the environment (prey, predator, and obstacle conditions) than if the motion path did not appear to be influenced by the environment (irrelevant condition) or if no environmental features were visible (none condition). Conversely, Experiments 2 and 3 showed that targets were least likely to be seen as animate when they appeared to respond passively to their environments - that is, only by the transfer of physical momentum.

Our experiments provide support for the intentionality hypothesis. To appreciate this, recall that the static foils in Experiment 1 never came into contact with the moving targets. Thus, our subjects must have inferred that the dot influenced the particle through a distance, since their ratings were affected by the dot's position. Influence without direct contact suggests that the influenced entity must have the abilities (1) to perceive through a distance, (2) to formulate goals based on what it perceives, and (3) to act based on its goals. This leads us to conclude that our subjects constructed intentional interpretations of the particle motion based on the location of the dot.

Our findings reinforce R. Gelman et al.'s (1995) claim that the perception of animacy is not due to motion alone. Our experiments also support their assertion that context affects the perception of animacy by influencing observers' inferences about the causes of motion. We find this 
assertion persuasive because it suggests a satisfying explanation of Stewart's (1982) finding that when subjects are shown displays of motions that violate energy conservation, they do not characterize all of these motions as animate; instead, they describe some as strange or artificial looking. ${ }^{11} \mathrm{R}$. Gelman et al. argue that these responses occur when observers fail to infer plausible causes - either mechanical or mental - for the motions.

Although our environmental manipulations had significant effects in all three experiments, the effect of context on animacy ratings was consistently small, especially compared with the effect of acceleration. It is unclear whether it was the simplistic nature of our displays, a tendency to weigh motion features more than contextual cues, or both, that was responsible for this result. Additional experiments using different backgrounds-for example, larger or smaller dots/paddles - could resolve this issue. It would also be interesting to modify our procedure or instructions to ensure that subjects assume the same level of magnification, since some of our subjects protested that it was unrealistic for living creatures to change direction as fast as the particles in our $80^{\circ}$-direction-change displays did, yet others rated those displays as definitely alive. Finally, additional research is needed to further identify the rules and constraints that govern observers' attempts to infer the causes of motions.

\section{Conclusions}

A recent article discussing animation techniques used to create films such as Toy Story, A Bug's Life, and Toy Story 2 (Porter \& Susman, 2000) reports that:

[Animators] understand that "lifelike" does not mean "has movement"; lifelike means "has a brain." The underlying notion of Pixar and Disney animation is that action is driven by the character's cognitive processes - that it reflects intelligence, personality, and emotion. The animator is constantly challenged to depict in an unmistakable yet compelling way that the brain is driving the action. ... Successful animators stay aware of their characters' motivations as they create their movements.

Our stimuli are certainly a far cry from the sophisticated animation sequences contained in Disney and Pixar films, but they suggest that an attribution of mental states to an agent is indeed instrumental in creating the impression that it is alive. We unconsciously seek to explain observed motion, and we attribute animacy when the explanation involves a mental cause.

In addition, our study makes progress toward identifying the specific conditions in which an object's motion yields an animate percept. We have replicated and extended previous work revealing that an extremely simple motion - namely, constant velocity except for a simultaneous speed increase and change in direction - can be sufficient. The experiments presented here suggest that this type of motion is effective in producing the perception of animacy because the movement lends itself to an explanation based on attributing an intention to the object. Although inanimate explanations of direction changes (bounces) and of speed changes (friction, gravity) exist, simultaneous changes in both speed and direction are unlikely to occur by chance. Thus, it is not surprising that, in the absence of supporting context, simultaneous changes in direction and increases in speed can convey an impression of volitional control, particularly when the direction changes and speed increases are relatively large. Whenever a simple supporting context is added, the impression of volition is augmented, resulting in significantly increased animacy ratings.

In summary, we propose that the perception of animacy arises whenever we attribute some minimal mental capacity, capable of supporting intentionality, to an object in the course of inferring the causes of its motion. This account represents an enhanced version of R. Gelman et al.'s hypothesis that animacy is perceived when observers infer that at least one of the causes of an object's motion is an intentional mental state. Our account predicts that the perception of animacy should be influenced by the environment, because the mental state of an entity generally depends on its context - that is, the physical features of the environment, as it perceives them-as well as the locations and assumed mental states of other entities. Our studies represent a step forward in understanding how human observers draw meaningful and causal interpretations of the world around them.

\section{REFERENCES}

BAssiLI, J. N. (1976). Temporal and spatial contingencies in the perception of social events. Journal of Personality \& Social Psychology, 33, 680-685.

Bingham, G. P., Rosenblum, L. D., \& Schmidt, R. C. (1995). Dynamics and the orientation of kinematic forms in visual event recognition. Journal of Experimental Psychology: Human Perception \& Performance, 21, 1473-1493.

Bonatti, L., Frot, E., Zangl, R., \& Mehler, J. (2002). The human first hypothesis: Identification of conspecifics and individuation of objects in the young infant. Cognitive Psychology, 44, 388-426.

Dasser, V., Ulbaek, I., \& Premack, D. (1989). The perception of intention. Science, 243, 365-367.

Dittrich, W. H., \& Lea, S. E. G. (1994). Visual perception of intentional motion. Perception, 23, 253-268.

Gelman, R., Durgin, F., \& Kaufman, L. (1995). Distinguishing between animates and inanimates: Not by motion alone. In D. Sperber, D. Premack, \& A. J. Premack (Eds.), Causal cognition: A multidisciplinary debate (pp. 150-184). Oxford: Oxford University Press, Clarendon Press.

Gelman, S. A., \& Gottfried, G. M. (1996). Children's causal explanations of animate and inanimate motion. Child Development, 67, 1970-1987.

Gergely, G., Nádasdy, Z., Csibra, G., \& Bíró, S. (1995). Taking the intentional stance at 12 months of age. Cognition, 56, 165-193.

Heider, F., \& Simmel, M. (1944). An experimental study of apparent behavior. American Journal of Psychology, 57, 243-259.

JoHnson, S. C. (2000). The recognition of mentalistic agents in infancy. Trends in Cognitive Sciences, 4, 22-28.

Johnson, S. C., Booth, A., \& O'Hearn, K. (2001). Inferring the goals of a nonhuman agent. Cognitive Development, 16, 637-656.

Kaiser, M. K., \& Proffitt, D. R. (1987). Observers' sensitivity to dynamic anomalies in collisions. Perception \& Psychophysics, 42, 275-280.

LEgerstee, M. (1994). The role of familiarity and sound in the development of person and object permanence. British Journal of Developmental Psychology, 12, 455-468.

LESLIE, A. M. (1984). Infant perception of a manual pick-up event. British Journal of Developmental Psychology, 2, 19-32.

Leslie, A. M. (1995). A theory of agency. In D. Sperber, D. Premack, 
\& A. J. Premack (Eds.), Causal cognition: A multidisciplinary debate (pp. 121-149). Oxford: Oxford University Press, Clarendon Press.

MANDLER, J. M. (1992). How to build a baby II: Conceptual primitives. Psychological Review, 99, 587-604.

Michotтe, A. (1950). Phenomenal permanence: Facts and theories. Acta Psychologica, 7, 298-322.

Michotтe, A. (1963). The perception of causality. New York: Basic Books.

Porter, T., \& Susman, G. (2000). Creating lifelike characters in Pixar movies. Communications of the ACM, 43, 25-29.

Premack, D. (1990). The infant's theory of self-propelled objects. Cognition, 36, 1-16.

Premack, D., \& Premack, A. J. (1995). Intention as psychological cause. In D. Sperber, D. Premack, \& A. J. Premack (Eds.), Causal cognition: A multidisciplinary debate (pp. 44-77). Oxford: Oxford University Press, Clarendon Press.

Schlottmann, A., \& Surian, L. (1999). Do 9-month-olds perceive causation-at-a-distance? Perception, 28, 1105-1113.

Stewart, J. A. (1982). Perception of animacy. Unpublished doctoral dissertation. University of Pennsylvania, Philadelphia.

Tremoulet, P. D., \& Feldman, J. (2000). Perception of animacy from the motion of a single object. Perception, 29, 943-951.

Van de Walle, G. A., Rubenstein, J. S., \& Spelke, E. S. (1998). Infant sensitivity to shadow motions. Cognitive Development, 13, 387-419.

WoODWARD, A. L. (1995, April). Infants' reasoning about the goals of a human actor. Poster session presented at the biennial meeting of the Society for Research in Child Development, Indianapolis, IN

Woodward, A. L., Phillips, A. T., \& Spelke, E. S. (1993). Infants expectations about the motion of animate versus inanimate objects. In Proceedings of the Fifteenth Annual Meeting of the Cognitive Science Society (pp. 1087-1091). Hillsdale, NJ: Erlbaum.

\section{NOTES}

1. Of course, no real motions violate Newtonian laws, apart from relativistic effects.

2 . In the case of a gradual deceleration, an invisible surface, causing friction, may be inferred, so even though this motion's visible component does not conserve energy, it may not produce a percept of animacy.

3. Conversations during postsession debriefings revealed that subjects sometimes inferred unseen goals and obstacles when viewing the displays used in our earlier studies, helping motivate the work reported here.
4. This finding concerned one reviewer, at whose suggestion we verified that each of our subjects used the entire 1-7 scale to rate our displays. Posttesting conversations with the subjects, as well as some comments made after the 10 practice trials, indicated that our subjects understood our instructions and that they interpreted the displays as intended (e.g., "the pouncing ones seemed most alive").

5. This exceeds the maximum amount of postcollision directional deviation that was tolerated as "natural" by Kaiser and Proffitt's (1987) subjects (deviations greater than $20^{\circ}$ were perceived to be anomalous). Thus, our subjects should have been able to detect that trajectories in the skew condition violated the laws governing inanimate collisions.

6. In previous studies (R. Gelman et al., 1995; Stewart, 1982), when round dots moved directly toward larger stationary objects in the background, observers tended to interpret the stationary objects as goal destinations - but these subjects were not instructed to think of the dots as microscopic particles.

7. A similar explanation was offered by an anonymous reviewer, who suggested that mental hysteresis may have led our subjects to assume that all of the displays involved the same two actors participating in different actions.

8. Our ratings scheme confounds anomalous and inanimate responses; however, postsession conversations with our subjects and free responses collected by Stewart (1982) after she showed similar displays to her subjects support the conclusions that our subjects perceived bounce displays with no speed change to be inanimate and that they inferred that particles whose trajectories looked anomalous were inanimate. Thus, the remainder of the discussion assumes that this is the correct interpretation of our subjects' ratings.

9. We thank an anonymous reviewer for pointing this out.

10. The idea that unexpectedly large increases in speed serve as a strong cue for animacy is consistent with Michotte's (1963) discovery that increasing the postcollision speed of one of the two blocks in his simple "launching" displays could alter observers' percept from physical causality to "triggering," which implies attributing animacy.

11. We were careful to instruct our subjects to give low animacy ratings if the particles' motions seemed odd or unnatural because we did not use free responses in our experiments.

(Manuscript received June 2, 2004; revision accepted for publication September 22, 2005.) 